\title{
Smoking, smoking cessation, and 7-year mortality in a cohort of Thai adults
}

\author{
Jiaying Zhao ${ }^{1}$, Cha-aim Pachanee ${ }^{2}$, Vasoontara Yiengprugsawann ${ }^{1 *}$, Sam-ang Seubsman ${ }^{1,3}$, Adrian Sleigh ${ }^{1}$ and Thai \\ Cohort Study Team
}

\begin{abstract}
Background: Smoking is a strong risk factor for mortality in both the developed and the developing world. However, there is still limited research to examine the impact of smoking cessation and mortality in middle-income Southeast Asian populations.

Methods: We use longitudinal data from a large Thai cohort of adult Open University students residing nationwide, linked with official death records to assess the association of smoking status and mortality risks during a 7-year follow-up. The log-rank test was used to evaluate the statistical probability of differential survival according to baseline smoking status. Multivariate hazard ratios (HR) were reported for smoking status and all-cause and cause-specific mortality.

Results: From 2005 baseline to 2012, current smokers were more likely to die than cohort members who ceased smoking and never smokers (1.9 vs 1.3 vs $0.6 \%, p<0.05$ ). The hazard of all-cause mortality increased with the daily amount of cigarette consumption among both current and former smokers. Cause of death analyses showed that current male smokers had a significantly increased risk of cardiovascular disease related mortality $(\mathrm{HR} 3.9[95 \% \mathrm{Cl}$ 1.8-8.1]). Former male smokers had a moderate increase in risk of dying from cardiovascular diseases compared to never smokers (HR 1.6 [95 \% Cl 0.7-3.4]). Current male smokers between 2005 and 2009 experienced highest subsequent mortality hazards during the period 2009-2012 compared to never smokers (HR 2.1 [95 \% Cl 1.4-3.4]). The higher risk of dying reduced if people quit smoking during the 2005-2009 follow-up period (HR 1.5 [95 \% Cl 0.7-3.3]). Risk for mortality fell even further among long-term quitters (HR 1.4 [95 \% Cl 0.9-2.2]).
\end{abstract}

Conclusion: Among a large nationwide cohort of Thai adults, current smokers were at a significantly and substantially higher risk of all-cause mortality, especially cardiovascular-related mortality. The higher risk of dying fell if people quit smoking and the risk for mortality was even lower among long-term quitters. Promotion of smoking cessation will contribute substantially to the reduction in avoidable mortality in Thailand.

Keywords: Smoking, Tobacco, Cessation, Mortality, Cardiovascular diseases, Thailand

\section{Introduction}

Smoking is a prominent risk factor of mortality in both the developed and the developing world [1-3]. In 2010, tobacco smoking was the second-leading risk factor for global burden of diseases $[4,5]$. Notably, a 50 -year prospective study of British doctors documented a secular improvement in longevity for never smokers, but not for

\footnotetext{
* Correspondence: vasoontara.yieng@anu.edu.au

${ }^{1}$ National Centre for Epidemiology and Population Health and Global Health Division, Research School of Population Health, The Australian National University, Canberra, Australia

Full list of author information is available at the end of the article
}

men who continued smoking cigarettes [6]. Moreover, among smokers, heavy smokers had higher mortality risk $[6,7]$. Similar results were found from 20-year follow-up in the Nurses' Health Study [8].

Previous literature has shown that smoking cessation can reduce overall mortality risk [9-12] and smoking cessation can also lower mortality from specific causes such as cancer $[13,14]$, respiratory diseases [15, 16], cardiovascular disease [17-19], and injury $[20,21]$. However, due to racial, cultural, and smoking behavior differences (e.g., age of initiation, duration), smoking and cessation patterns may differ 
across populations. Therefore, local mortality data in relation to smoking cessation are essential to understand potential population benefits for public health campaigns to promote smoking reduction.

Mortality risks decrease with the length of time since quitting smoking; in the UK, for example, it was estimated that quitting smoking at age $60,50,40$, or 30 gained about $3,6,9$, or 10 years of life expectancy, respectively [7]. A large population-based Japanese cohort study underway since 1950 showed that for heavy smokers, life expectancy was reduced by almost a decade (8 years for men and 10 years for women) [22]. To date, in Asia, most studies of smoking cessation and mortality have been among more affluent East Asian countries [12, 13, 17, 19]. There is limited research to date examining how quickly the benefits of smoking cessation can be observed after quitting smoking in middle-income Southeast Asian populations.

Thailand, a developing country in Southeast Asia, has undergone rapid economic growth and a health-risk transition in recent decades, moving from malnutrition and infectious diseases to chronic diseases as major causes of death. In 2009, smoking contributed to the highest death rate and was also the second-leadingrisk factor contributing to burden of disease among Thai males [23]. Smoking-related deaths (lung cancer, heart disease, chronic obstructive pulmonary disease) increased from 45,136 cases in 2004 to 50,710 cases in 2009 [23]. The overall smoking rate in Thailand had decreased from $32.0 \%$ in 1991 to $19.9 \%$ in 2013 and $20.7 \%$ in 2014 [24]. A recent Global Adult Tobacco Survey suggested that Thailand experienced a significant decline in the percentage of smokers who made a quit attempt during the past 12 months from 2009 (49.8\%) to 2011 (36.7\%) [25]. Almost all adults (>97.0 \%) believed that smoking caused serious illness and lung cancer but fewer (around $80 \%$ ) thought smoking caused stroke and heart attack. This may be partly because local evidence of smoking related to specific diseases and benefits of quitting smoking is still rare.

We use data from a large Thai cohort made up of adult Open University students residing nationwide, linked with official death records to assess the association with reported smoking status and mortality risks within seven year follow-up. We present the relationship between smoking status, including average number of cigarettes smoked daily, and subsequent all-cause and cause-specific mortality. We compare mortality risk among never smokers, long-term quitters (quit more than 4 years), new quitters (quit within 4 years), and current smokers. Our findings provide empirical evidence on smoking cessation and mortality in middle-income Southeast Asia.

\section{Methods}

\section{Study population}

We use the Thai Cohort Study (TCS) data, which collected information on health status as well as risk and protective factors associated with socioeconomic development. The study included 87,151 adult distancelearning students enrolled at Sukhothai Thammathirat Open University, residing all over Thailand in 2005. The participants represented the Thai population well in terms of social geography, religion, socioeconomic status, and income $[26,27]$. TCS participants were followed up in 2009 by mail and 60,569 participants completed questionnaires for an overall response rate of $71 \%$ [28].

TCS data were linked to official mortality data from the Thai Ministry of Interior using the 13-digit citizen identification number, a unique ID for each Thai person. Up until August 15, 2012, 767 deaths were recorded among the cohort members. The coverage of registration of adult deaths in Thailand was $86 \%$ from 1950 to 2000 [29], which improved further to $95 \%$ over the period reported in this study [30].

Cause-of-death information occurring before the end of $2010(n=583)$ was provided and verified by the Ministry of Public Health. Causes of deaths which were ill-defined had been investigated by the vital statistics office of the Ministry of Public Health according to hospital records and verbal autopsies. Among our analyses, ill-defined deaths were only $8.4 \%(n=49)$ of the total number of deaths in the cohort.

\section{Measurements}

Smoking status in 2005 and 2009 was categorized into never smoker, former smoker, or current smoker. In 2005, both current and former smokers were asked to note average number of cigarettes smoked daily. We further categorized smoking status in 2005 into never smoker, former light smokers (number of cigarettes $<10$ ), former medium smokers (number of cigarettes 10-19), former heavy smokers (number of cigarettes $>=20$ ), current light smokers (number of cigarettes <10), current medium smokers (number of cigarettes 10-19), current heavy smokers (number of cigarettes $>=20$ ). For 4 -year smoking status, we combined dynamic smoking status from 2005 to 2009 into four categories: never smoker (2005-09), those who had stopped smoking from 2005, those who had stopped smoking in 2009, and current smoker in 2005 and 2009.

Analyses included baseline data on sex, birth year, urban or rural residence, income, and health insurance coverage. Personal monthly income measured in 2005 was classified as $\leq 3000,3001-20,000$, or $>20,000$ Baht (1 \$US 30 Baht). Household monthly income measured in 2009 was classified as $<7000,7001-30,000$, or $>30,000$ Baht. Health insurance is provided through three 
programs: the civil servant scheme, the Universal Coverage scheme available to Thai nationals (initially called the 30 Baht scheme), and others including social security for private employees.

Potential confounders related to health behaviors including alcohol drinking and physical activity in 2005 and 2009 were also analysed. We classified alcohol consumption in 2005 as occasional social drinker, never drinker, current regular drinker, or now stopped. In 2009 , the categories included non-drinker, light drinker ( $\leq 7$ glasses per week), or moderate or heavy drinker $(\geq 8$ glasses per week). We recoded weekly physical activity in 2005 and 2009 as less than 7 sessions or 7 sessions or more, based on standard measures from the International Physical Activity Questionnaire and Active Australia Survey [31].

Self-rated health was used to as an indicator of overall general health status at baseline, assessed with a standardized question, "Overall, how would you rate your health over the last 4 weeks?" in both 2005 and 2009. Responses were categorized as excellent, very good, good, fair, poor, and very poor. We divided selfrated health into positive (excellent/very good/good/ fair) and negative (poor and very poor) groups. Body mass index (BMI) in 2005 and 2009 was based on self-reported height and weight. We used Asian BMI standards: underweight $(<18.5)$, normal $(18.5$ to $<23)$, overweight at risk $(23$ to $<25)$, obese I $(25$ to $<30)$, and obese II $(\geq 30)$ [32]. In a separate study, selfreported height and weight was validated and showed the estimates were reasonably accurate and suitable for use in a large cohort study [33].

\section{Statistical analyses}

We examined the distribution of cohort smoking status in 2005 by potential confounders (e.g., main sociodemographic attributes, risk behaviors, and physical activity). We examined the distribution of smoking by survival status on December 31, 2010 and August 15, 2012. For males we also assessed the distribution of smoking status, including the information on the daily average number of cigarettes smoked in 2005 by survival status. Kaplan Meier survival curves display differential survival patterns by baseline self-rated health (March 1, 2005). End-point events were all-cause deaths and cause-specific deaths until December 31, 2010 (cardiovascular diseases, cancers, injury, and other causes) and all-cause deaths until August 15, 2012. We used the log-rank test to test the statistical probability of observed difference in survival patterns according to 2005 smoking status.

As males constituted the vast majority of smokers in the cohort [34], multivariate regression analyses only focused on males. Multivariate hazard ratios (HR) and $95 \%$ confidence intervals for mortality by baseline
(2005) smoking status were estimated using the Cox model after confirming that the assumption of proportionality of hazards was held. For each of four specific death outcomes (cardiovascular diseases, cancers, injury, and other causes), models were developed in the same way as for all-cause mortality.

We performed separate Cox regression analyses examining all-cause mortality in the cohort between 2009 and 2012 in relation to longitudinal dynamic smoking status (2005-2009). We used 'never smoker' as a reference. The end-point events for those who were followed up in 2009 were all-cause deaths until August 15, 2012. Covariates include birth year and health insurance coverage in 2005 , as well as other four-year follow-up information (2009) including residence, monthly household income, drinking, physical activity, body mass index, and selfreported overall health.

\section{Ethical approval}

Informed written consent was obtained from all participants. All students were advised that they could withdraw, or not participate, without any effect on their academic progress. The questionnaires never sought sensitive personal information and no biological samples were taken. Ethics approval was obtained from Sukhothai Thammathirat Open University Research and Development Institute (protocol 0522/ 10) and the Australian National University Human Research Ethics Committee (protocols 2004/344 and 2009/570).

\section{Results}

\section{Distribution of smoking by potential confounders}

Smoking experience was reported frequently among males and infrequently among females (Table 1). The proportion of females who never smoke was more than $90 \%$ in the cohort.

Among males, more than $90 \%$ of cohort members were born after 1960 (aged $\leq 45$ years in 2005 baseline). Never smokers had a higher proportion of younger birth cohort (born 1980 or after, aged $\leq 25$ years in 2005 baseline). The proportion of male current smokers were similar across birth cohorts - except for low proportions among the oldest (born before 1960) and the youngest (born in 1980 or after). Lower-income males were more likely to be current smokers, and less likely to quit smoking. The difference in smoking status between rural and urban males was modest. Males who reported being regular alcohol drinkers were more likely to be current smokers. Compared with never smokers, current smokers and former smokers had higher proportions reporting poor health. 
Table 1 Socio-demographic attributes at baseline (2005) by smoking status and sex in the Thai Cohort Study

\begin{tabular}{|c|c|c|c|c|c|c|c|c|c|c|}
\hline \multicolumn{2}{|c|}{ Socio-demographic attributes } & \multicolumn{9}{|c|}{ Column \% distribution of smoking status by sex } \\
\hline & & \multicolumn{3}{|c|}{ Total $(n=84,573)$} & \multicolumn{3}{|c|}{ Males $(n=38,450)$} & \multicolumn{3}{|c|}{ Females $(n=46,123)$} \\
\hline & & $\begin{array}{l}\text { Never } \\
(n=61,143)\end{array}$ & $\begin{array}{l}\text { Former } \\
(n=14,698)\end{array}$ & $\begin{array}{l}\text { Current } \\
(n=8732)\end{array}$ & $\begin{array}{l}\text { Never } \\
(n=17,653)\end{array}$ & $\begin{array}{l}\text { Former } \\
(n=12,542)\end{array}$ & $\begin{array}{l}\text { Current } \\
(n=8255)\end{array}$ & $\begin{array}{l}\text { Never } \\
(n=43,490)\end{array}$ & $\begin{array}{l}\text { Former } \\
(n=2156)\end{array}$ & $\begin{array}{l}\text { Current } \\
(n=477)\end{array}$ \\
\hline \multirow[t]{5}{*}{ Birth Year } & -1959 (> = 46 years) & 4.3 & 12.3 & 7.8 & 6.2 & 13.9 & 8.1 & 3.6 & 3.0 & 2.9 \\
\hline & 1960-1969 (36-45 years) & 18.3 & 28.4 & 24.5 & 20.4 & 30.2 & 24.7 & 17.4 & 17.9 & 21.4 \\
\hline & 1970-1974 (31-35 years) & 16.3 & 20.0 & 22.3 & 17.4 & 20.1 & 22.5 & 15.8 & 19.6 & 19.5 \\
\hline & 1975-1979 (26-30 years) & 25.7 & 22.6 & 27.0 & 25.3 & 21.2 & 26.8 & 25.9 & 30.6 & 31.7 \\
\hline & $1980-(<=25$ years $)$ & 35.4 & 16.7 & 18.3 & 30.7 & 14.6 & 18.0 & 37.4 & 28.9 & 24.5 \\
\hline \multirow{3}{*}{$\begin{array}{l}\text { Personal } \\
\text { monthly } \\
\text { income } \\
\text { (Baht) }\end{array}$} & $<3000$ & 11.4 & 8.7 & 12.4 & 13.1 & 8.7 & 12.6 & 10.6 & 8.2 & 8.4 \\
\hline & $3001-20,000$ & 79.3 & 75.7 & 76.3 & 74.5 & 74.6 & 76.4 & 81.3 & 81.9 & 75.6 \\
\hline & $>=20.001$ & 9.3 & 15.7 & 11.3 & 12.4 & 16.7 & 11.0 & 8.1 & 9.8 & 16.1 \\
\hline \multirow{2}{*}{$\begin{array}{l}\text { Place of } \\
\text { residence }\end{array}$} & Urban & 51.4 & 53.0 & 52.0 & 49.4 & 51.3 & 50.9 & 52.2 & 63.2 & 71.2 \\
\hline & Rural & 48.6 & 47.0 & 48.0 & 50.6 & 48.7 & 49.1 & 47.8 & 36.8 & 28.8 \\
\hline \multirow{3}{*}{$\begin{array}{l}\text { Health } \\
\text { Insurance } \\
\text { Coverage }\end{array}$} & 30 Baht scheme & 13.7 & 11.4 & 13.0 & 13.4 & 11.1 & 13.2 & 13.8 & 13.0 & 8.4 \\
\hline & Civil servant scheme & 23.2 & 31.4 & 27.2 & 29.0 & 34.1 & 28.0 & 20.8 & 15.8 & 12.4 \\
\hline & Others & 63.2 & 57.2 & 59.9 & 57.6 & 54.8 & 58.7 & 65.4 & 71.2 & 79.2 \\
\hline \multirow{4}{*}{$\begin{array}{l}\text { Alcohol } \\
\text { consumption }\end{array}$} & Occasional & 56.5 & 67.8 & 66.7 & 69.5 & 67.3 & 66.1 & 51.2 & 70.9 & 76.1 \\
\hline & Never & 35.6 & 3.2 & 3.3 & 19.7 & 2.8 & 3.3 & 42.1 & 5.8 & 3.6 \\
\hline & Regular & 1.3 & 11.5 & 18.6 & 3.7 & 12.9 & 18.8 & 0.4 & 3.7 & 14.0 \\
\hline & Stopped & 6.6 & 17.4 & 11.4 & 7.1 & 17.1 & 11.7 & 6.3 & 19.6 & 6.3 \\
\hline \multirow{2}{*}{$\begin{array}{l}\text { Weekly } \\
\text { physical } \\
\text { activity }\end{array}$} & $<7$ sessions & 35.9 & 29.2 & 27.3 & 24.4 & 26.5 & 26.1 & 40.6 & 45.0 & 49.4 \\
\hline & $7+$ sessions & 64.1 & 70.8 & 72.7 & 75.6 & 73.5 & 73.9 & 59.4 & 55.0 & 50.6 \\
\hline \multirow{5}{*}{$\begin{array}{l}\text { Body mass } \\
\text { index }\end{array}$} & Underweight(<18.5) & 17.9 & 6.2 & 6.9 & 7.6 & 4.1 & 5.9 & 22.1 & 18.4 & 22.7 \\
\hline & Normal (18.5 to <23) & 57.0 & 46.4 & 49.2 & 53.1 & 44.6 & 49.0 & 58.6 & 56.7 & 52.2 \\
\hline & $\begin{array}{l}\text { Overweight at risk } \\
(23 \text { to }<25)\end{array}$ & 12.7 & 22.1 & 20.4 & 20.3 & 24.1 & 20.8 & 9.6 & 10.4 & 12.1 \\
\hline & Obesity I (25 to <30) & 10.1 & 21.9 & 20.3 & 16.2 & 23.8 & 21.0 & 7.6 & 11.0 & 8.5 \\
\hline & Obesity II ( $\geq 30)$ & 2.3 & 3.4 & 3.3 & 2.8 & 3.4 & 3.2 & 2.1 & 3.6 & 4.5 \\
\hline \multirow{2}{*}{$\begin{array}{l}\text { Poor self- } \\
\text { reported } \\
\text { health }\end{array}$} & No & 95.5 & 95.0 & 95.4 & 96.9 & 95.6 & 95.6 & 95.0 & 91.4 & 91.4 \\
\hline & Yes & 4.5 & 5.0 & 4.6 & 3.1 & 4.4 & 4.4 & 5.0 & 8.6 & 8.6 \\
\hline
\end{tabular}

\section{Smoking and mortality}

From baseline (2005) to 2012, current smokers were more likely to die than people who quit smoking and those who never smoked (1.9 vs 1.3 vs $0.6, p<0.05)$ (Table 2). The patterns of association between smoking status and mortality risk for males were similar to those for the whole cohort. For males, former heavy smokers (2.5\%) were more likely to die compared with former medium smokers (1.7 \%) and former light smokers (0.9 \%). A similar trend for heavy, medium and light smokers was observed among current smokers (2.7 vs 2.2 vs $1.5 \%, p<0.05)$.

There were 78 cohort deaths from cardiovascular diseases, 118 deaths from cancers, 204 from injury, and 183 deaths from other causes of death from baseline in 2005 up to 2010. Cause-specific analyses suggested that current smokers for both total population and males were more likely to die from cardiovascular diseases (CVD), injury, and from other causes $(p<0.05)$ than those who had never smoked. In addition, current smokers among the whole cohort and particularly males also had a higher risk of dying from cardiovascular diseases and injury than former smokers $(p<0.05)$. However, the risk of dying from cancers across different groups by smoking status was not significant.

Kaplan-Meier survival curves by smoking status (Fig. 1) indicated that current smokers had a higher risk of allcause and cause-specific deaths (i.e., cardiovascular diseases, cancer, injury, and other causes of death) than never smokers (log-rank test $p<0.05$ ). Cohort members who stopped smoking also reduced the risk of mortality from all-cause mortality, cardiovascular diseases, and 
Table 2 Survival data by smoking status (row percent) for the Thai Cohort Study, 2005-2012

\begin{tabular}{|c|c|c|c|c|c|c|c|c|c|}
\hline \multirow{4}{*}{ Sex } & \multirow{4}{*}{ Smoking status (2005) } & \multicolumn{8}{|c|}{ End-point follow up (row \%) } \\
\hline & & \multirow{2}{*}{\multicolumn{2}{|c|}{$\begin{array}{l}\text { August 15, } 2012 \\
\text { Survival status }\end{array}$}} & \multicolumn{6}{|c|}{ December 31, 2010} \\
\hline & & & & \multicolumn{2}{|c|}{ Survival status } & \multicolumn{4}{|c|}{ Deaths by causes } \\
\hline & & Survived & Died & Survived & Died & Cardiovascular & Cancer & Injury & Other \\
\hline \multirow[t]{4}{*}{ Total } & Never & 60,758 & $394(0.6 \%)$ & 60,848 & $304(0.5 \%)$ & $31(0.1 \%)$ & $68(0.1 \%)$ & $115(0.2 \%)$ & $90(0.1 \%)$ \\
\hline & Former & 14,513 & $189(1.3 \%)$ & 14,563 & 139 (0.9 \%) & $20(0.1 \%)$ & $32(0.2 \%)$ & $35(0.2 \%)$ & $52(0.4 \%)$ \\
\hline & Current & 8569 & $163(1.9 \%)$ & 8608 & $124(1.4 \%)$ & $25(0.3 \%)$ & $16(0.2 \%)$ & $49(0.6 \%)$ & $34(0.4 \%)$ \\
\hline & Total & 83,840 & $746(0.9 \%)$ & 84,019 & $567(0.7 \%)$ & $78(0.1 \%)$ & 118 (0.1\%) & $204(0.2 \%)$ & $183(0.2 \%)$ \\
\hline \multirow[t]{10}{*}{ Males } & Never & 17,477 & $179(1.0 \%)$ & 17,517 & $139(0.8 \%)$ & $16(0.1 \%)$ & $23(0.1 \%)$ & $63(0.4 \%)$ & $37(0.2 \%)$ \\
\hline & Former All & 12,369 & $174(1.4 \%)$ & 12,414 & $129(1.0 \%)$ & $19(0.2 \%)$ & $28(0.2 \%)$ & $33(0.3 \%)$ & $49(0.4 \%)$ \\
\hline & Former light smokers & 7011 & $64(0.9 \%)$ & 7026 & $49(0.7 \%)$ & $7(0.1 \%)$ & $7(0.1 \%)$ & $16(0.2 \%)$ & $19(0.3 \%)$ \\
\hline & Former medium smokers & 3197 & $54(1.7 \%)$ & 3211 & $40(1.2 \%)$ & $5(0.2 \%)$ & $10(0.3 \%)$ & $8(0.3 \%)$ & $17(0.5 \%)$ \\
\hline & Former heavy smokers & 2161 & $56(2.5 \%)$ & 2177 & $40(1.8 \%)$ & $7(0.3 \%)$ & $11(0.5 \%)$ & $9(0.4 \%)$ & $13(0.6 \%)$ \\
\hline & Current All & 8098 & $157(1.9 \%)$ & 8136 & 119 (1.4\%) & $24(0.3 \%)$ & $14(0.2 \%)$ & $49(0.6 \%)$ & $32(0.4 \%)$ \\
\hline & Current light smokers & 3886 & $57(1.5 \%)$ & 3902 & $41(1.0 \%)$ & $6(0.2 \%)$ & $4(0.1 \%)$ & $21(0.5 \%)$ & $10(0.3 \%)$ \\
\hline & Current medium smokers & 3035 & $67(2.2 \%)$ & 3048 & $54(1.7 \%)$ & $12(0.4 \%)$ & $8(0.3 \%)$ & $18(0.6 \%)$ & $16(0.5 \%)$ \\
\hline & Current heavy smokers & 1177 & $33(2.7 \%)$ & 1186 & $24(2.0 \%)$ & $6(0.5 \%)$ & $2(0.2 \%)$ & $10(0.8 \%)$ & $6(0.5 \%)$ \\
\hline & Total & 37,944 & $510(1.3 \%)$ & 38,067 & $387(1.0 \%)$ & $61(0.2 \%)$ & $65(0.2 \%)$ & $150(0.4 \%)$ & $123(0.3 \%)$ \\
\hline
\end{tabular}

injury compared with current smokers. However, mortality from cancers and other causes of death did not significantly differ between current smokers and former smokers. The survival patterns for males were similar to those among the whole cohort (Fig. 2).

As there were very few female deaths among current smokers $(n=6)$ and former smokers $(n=15)$, the analyses of the Cox hazard model are only conducted for males. In the Cox proportional hazard model, current smokers at baseline, after controlling for age, rural or urban residence in 2005, monthly income, alcohol drinking, physical activity, body mass index, and self-reported health had a significantly higher risk of subsequent death till 2012 (HR 1.8 [95 \% CI:1.4-2.3]) than never smokers (Table 3). Former smokers also had a higher risk of mortality than never smokers, but this effect was not significant. The hazard ratio of premature mortality for former smokers was much lower than that of current smokers (HR 1.2 [95 \% CI:0.9-1.5]).

There was an increasing trend in mortality risk associated with daily cigarette consumption. The hazard ratio of mortality for former heavy smokers compared with never smokers (HR 1.7 [95 \% CI 1.2-2.5]) was higher than that of former light smokers (HR 0.9 [95 \% CI 0.7-1.2]) and former medium smokers (HR 1.5 [95 \% CI 1.0-2.1]). Similarly among current smokers, the hazard ratios of premature mortality compared with never smokers were highest for heavy smokers (HR 2.4 [95 \% CI 1.6-3.6]), followed by medium smokers (HR 1.9 [95 \% CI 1.4-2.7]) and light smokers (HR 1.6 [95 \% CI 1.2-2.2]).
Cause of death analyses showed that current male smokers had a significantly increased risk of death from cardiovascular disease (HR 3.9 [95 \% CI:1.8-8.1]) after adjusting for demographic factors, behavioral variables, body mass index, and self-reported overall health. Current smokers also had a higher mortality risk for deaths from injury (HR 1.7 [95 \% CI 1.1-2.6]) and from other causes (HR 1.8 [95 \% CI 1.1-3.0]) after adjusting for potential covariates. Former smokers had a lower risk of dying from cardiovascular diseases (HR 0.4 [95 \% CI 0.2-0.8]) and from injury (HR 0.5 [95 \% CI $0.3-0.7]$ ) than current smokers, though former smokers had a moderate increase in risk for dying from cardiovascular deaths compared to those who never smoked (HR 1.6 [95 \% CI 0.7-3.4]). For other causes of death, effects of smoking were not significant comparing mortality risk for former smokers and current smokers (HR 0.9 [95\% CI 0.6-1.4]), but we noted that former smokers had a moderately higher risk than never smokers (HR 1.6 [95 \% CI 1.0-2.6]). There was no significant increase in risk of cancer mortality among current smokers or former smokers compared with never smokers. We did not conduct analysis on association between the daily consumption of cigarettes and cause-specific mortality risk in the Cox hazard model as there were very few deaths in each category.

The longitudinal smoking status during the 20052009 period was associated with subsequent survival between 2009 and 2012 (Table 4). For males, never smokers were more likely to survive from 2009 to 2012. After full adjustment for demographic characteristics, 

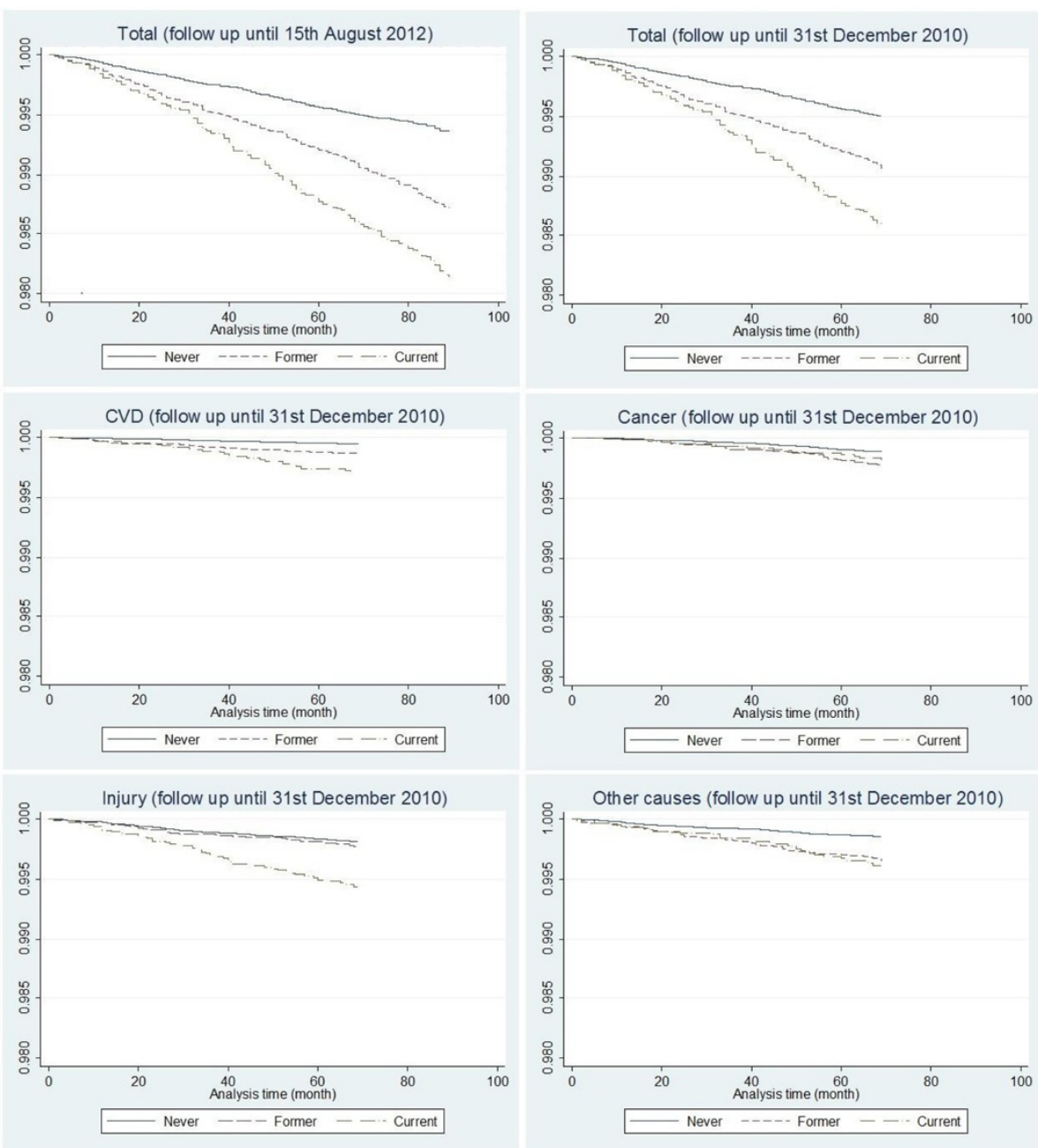

Fig. 1 Kaplan-Meier survival curves for smoking status (2005) by cause of death. Note: $y$ axis records the proportion surviving; $x$ axis records the duration of survival in month

risk behaviors, and physical activity in 2009, current smokers between 2005 and 2009 experienced highest mortality hazards on the next 4 years compared to never smokers (HR 2.1 [95 \% CI 1.4-3.4]). The risk of dying reduced if people quit smoking during the 2005-2009 period (HR 1.5 [95 \% CI 0.7-3.3]). Risk of mortality was even lower among long-term quitters (HR 1.4 [95\% CI $0.9-2.2])$.

\section{Discussion}

We report on smoking and mortality from 5- and 7-year follow up of a large cohort of Thai adults. Smoking was almost exclusively a health risk for males because very few Thai females smoke. Among males, more than $90 \%$ of cohort members were born after 1960 (aged $\leq 45$ years in 2005 baseline). Never smokers had a higher proportion of the younger birth cohort (aged $\leq 25$ years in 2005 baseline). Current smokers in the cohort were more likely to die from all-cause mortality than former smokers or non-smokers. The hazard of all-cause mortality compared to non-smokers increased with the daily amount of cigarette consumption among both current and former smokers. Cause of death analysis showed that current male smokers had a significantly higher risk of cardiovascular disease mortality (HR 3.9 [95 \% CI 1.8-8.1]). Current male smokers in both 2005 and 2009 experienced highest subsequent all-cause mortality hazards (HR 2.1 [95 \% CI 1.4-3.4]). The risk of dying reduced if people quit smoking, and mortality risk was even lower among long-term quitters.

Our findings confirm the smoking and mortality relationship previously reported in Western countries $[1,3]$. Our findings also show that mortality risk increased with the daily amount smoked among both former and current smokers in the Thai population, as has been reported in the West [7]. Our study 

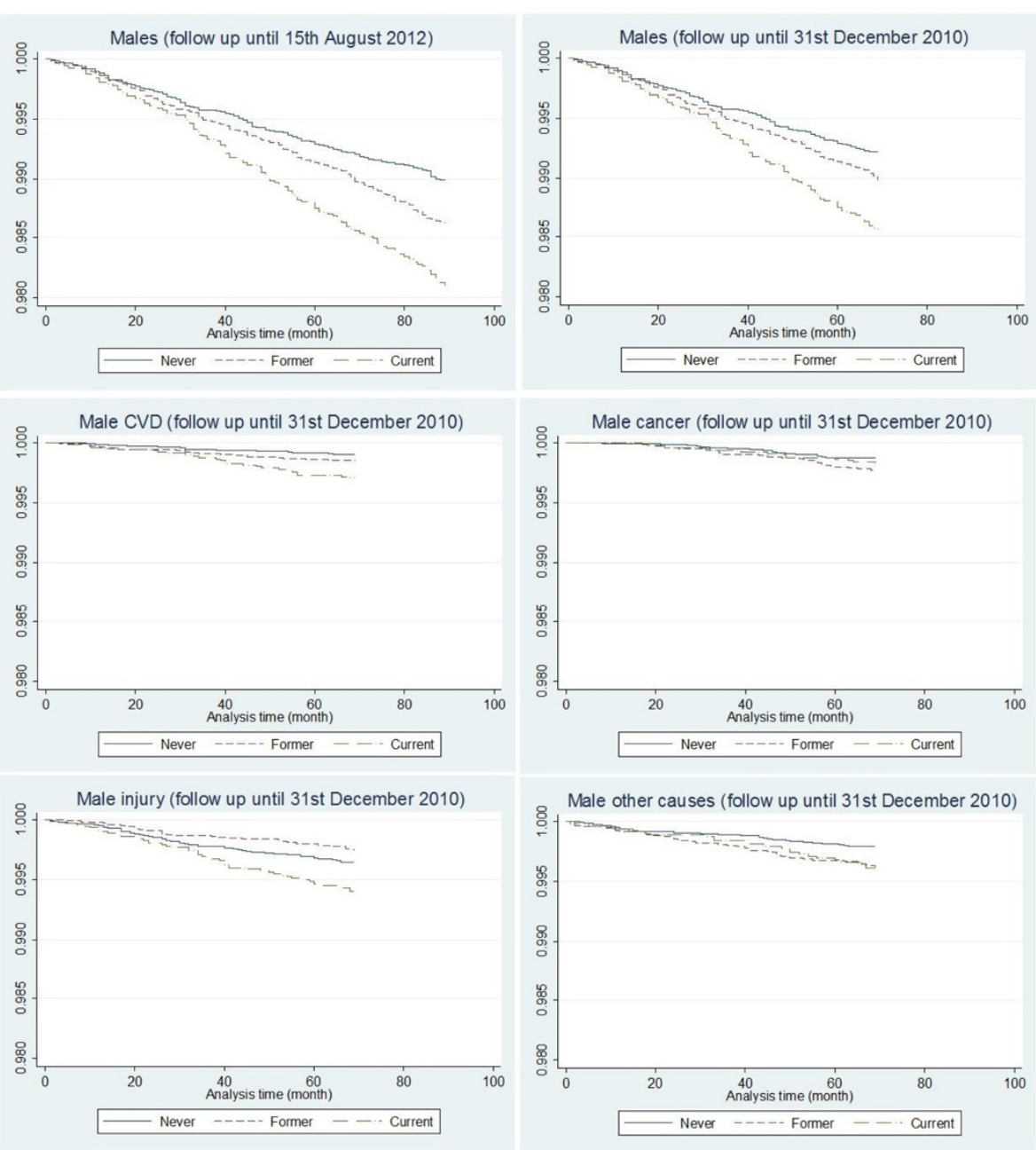

Fig. 2 Kaplan-Meier survival curves for smoking status (2005) by cause of death, males. Note: $y$ axis records the proportion surviving; $x$ axis records the duration of survival in month

Table 3 Mortality and smoking status (2005) for males in the Thai Cohort Study, 2005-2012

\begin{tabular}{|c|c|c|c|c|c|c|}
\hline \multirow[t]{2}{*}{ Smoking status (2005) } & \multirow{2}{*}{$\begin{array}{l}\text { All-causes } \\
\text { (to Aug 2012) }\end{array}$} & \multirow{2}{*}{$\begin{array}{l}\text { All causes } \\
\text { (till Dec 2010) }\end{array}$} & \multicolumn{4}{|l|}{ Causes of death } \\
\hline & & & Cardiovascular & Cancer & Injury & Other \\
\hline Former & $1.2[0.9-1.5]$ & $1.1[0.9-1.5]$ & $1.6[0.7-3.4]$ & $1.0[0.5-1.8]$ & $0.8[0.5-1.3]$ & $1.6[1.0-2.6]$ \\
\hline Current & $1.8[1.4-2.3]$ & $1.8[1.3-2.3]$ & $3.9[1.8-8.1]$ & $0.9[0.4-2.0]$ & $1.7[1.1-2.6]$ & $1.8[1.1-3.0]$ \\
\hline Former light smokers & $0.9[0.7-1.2]$ & - & - & - & - & - \\
\hline Former medium smokers & $1.5[1.0-2.1]$ & - & - & - & - & - \\
\hline Former heavy smokers & $1.7[1.2-2.5]$ & - & - & - & - & - \\
\hline Current light smokers & $1.6[1.2-2.2]$ & - & - & - & - & - \\
\hline Current medium smokers & $1.9[1.4-2.7]$ & - & - & - & - & - \\
\hline Current heavy smokers & $2.4[1.6-3.6]$ & - & - & - & - & - \\
\hline
\end{tabular}

Hazard ratios compare mortality over the follow-up period by baseline smoking status (reference category 'never smoker' at baseline in 2005). Models adjusted for birth year, monthly income, urban or rural residence in 2005 , health insurance coverage, alcohol drinking, physical activities, body mass index, and self-reported health 
Table 4 Smoking (2005-09) and subsequent outcomes (2009-12) for males in the Thai Cohort Study: survival and mortality

\begin{tabular}{|c|c|c|c|}
\hline \multirow[t]{3}{*}{ Longitudinal smoking status (2005-09) } & \multicolumn{2}{|c|}{$2012 \%$ Survival by smoking status } & \multirow{3}{*}{$\begin{array}{l}\text { Hazard ratios }(95 \% \text { Cl) for mortality } \\
\text { from } 2009 \text { to } 2012 \text { by smoking status }\end{array}$} \\
\hline & Survived & Died & \\
\hline & $(n=22,026)$ & $(n=161)$ & \\
\hline Never smoke & 99.5 & 0.5 & Reference \\
\hline Quit smoking from 2005 & 99.1 & 0.9 & $1.4[0.9-2.2]$ \\
\hline Current smoking in 2005 and quit in 2009 & 99.1 & 0.9 & $1.5[0.7-3.3]$ \\
\hline Current smoking in 2005 and in 2009 & 98.9 & 1.1 & $2.1[1.4-3.4]$ \\
\hline
\end{tabular}

Hazard ratios compare mortality over the follow-up period by longitudinal smoking status over the previous period (reference category 'never smokers' in both 2005 and 2009). The model includes age category (five groups), monthly household income (2009), urban or rural residence in 2009, health insurance coverage (2005), alcohol drinking (2009), physical activities (2009), body mass index (2009), and self-reported health (2009)

further provides longitudinal nationwide evidence supporting various reports from Thailand, including the important adverse effects of smoking among the Thai population as shown by the Burden of Disease study in 2009 [35]. Our study reveals a strong association between smoking and cardiovascular death in young to middle age Thais, which is consistent with the results of other studies [36]. Our study further confirms that cessation of smoking among Thais can lower mortality from specific causes such as cardiovascular diseases [17-19]. In addition, association between smoking and injury has been noted in other studies [20,21]. Plausible scenarios include fires or road crashes caused by smoking when falling asleep, distracted, or inattentive, though the demographic and psychological characteristics of smokers may be confounders. Our findings also support a previous study on smoking and mortality among rural Thais, especially among men [37] and provide additional evidence on the benefits of smoking cessation on cause-specific mortality.

Compared to Western countries, female smoking is particularly low in Thailand $[34,38]$. This is an enormous advantage, for half the Thai population could avoid exposure to one of the deadliest substances available publicly. In another setting current female smokers, compared to female non-smokers, had double all-cause mortality rates and triple stroke and cerebrovascular deaths [39]. Furthermore, smoking cessation among females reduced all-cause deaths by $25 \%$ and cardiovascular deaths by $60 \%$ [8]. However, trends in female smoking in some middle- and low-income countries are increasing [40]. Hence, countries with low rates among females should actively support such culturally-conferred public health advantage enjoyed by the population.

The main strength of our study is its large participation by Thai adults who responded to a comprehensive baseline questionnaire addressing a wide array of social and health characteristics. This information, combined with longitudinal analyses, allows elimination of many confounders and this helps to establish causal pathways such as the link between smoking and mortality. Our cohort members share certain important characteristics with the general Thai population including similar modest incomes, similar geographic distribution, and similar ethnicity and religion. Cohort members have completed high school education, which could have positive influence on the uptake of health promotion including smoking cessation [41]. A study by Pachanee found the advantage of education in reducing smoking, after adjusting for other factors [42]. Given relatively higher education among cohort members than general Thais and associated lower health risks and better outcomes, our findings confirm the importance of intensifying the campaign on smoking control, with a clear focus of reducing male smoking and preventing female smoking.

We acknowledge some limitations of our study as follows. First, cohort members were not asked about reasons for smoking cessation and some could be due to health reasons. We used self-reported health as a proxy for overall health in our analyses. We also conducted a sensitivity analysis to test similar models for all-cause mortality with Cox regression but excluded cohort members who reported chronic diseases (e.g., cancers, ischemic heart diseases, cerebrovascular diseases, and diabetes) at the 2005 baseline. The following HRs by smoking status (1.3 [95 \% CI 1.0-1.6] for former smokers and 1.8 [95 \% CI 1.4-2.4] for current smokers) during the 7-year follow up (up to August 2012) from this sensitivity analysis were similar to the corresponding figures (1.2 [95 \% CI 0.9-1.5] for former smokers and 1.8 [95 \% CI 1.4-2.3] for current smokers) in the Results section. The models in the sensitivity analysis for causespecific outcomes only excluded people who reported certain diseases at the 2005 baseline which could be related to the specific cause of deaths. For example, for analysis of cancer deaths, cohort members who reported cancers at the 2005 baseline were excluded. For analysis of CVD mortality, we excluded cohort members with ischemic heart disease, cerebrovascular disease, and diabetes from the 2005 baseline. For all other causes of death, we restricted to cohort members who did not report diabetes in 2005. The patterns of HR for smoking status from the sensitivity analysis were similar to those reported in the Results section. However, the HRs for 
CVD mortality in the sensitivity analysis $(2.5$ [95 \% CI 1.0-6.3] for former smokers and 5.1 [95 \% CI 2.1-12.4] for current smokers) were higher than the corresponding figures reported in the Results section (1.6 [95 \% CI $0.7-3.4$ ] for former smokers and 3.9 [95 \% CI 1.8-8.1] for current smokers).

We also note possible limitation of the study relating to the lack of information on types of tobacco smoked in our study; this could be important if mortality risk depends on tobacco type. However, a recent longitudinal study in rural Thailand noted that there was no statistical difference in mortality risk between hand-rolled and manufactured tobacco [37]. Nevertheless, information on types of tobacco used could be helpful for designing interventions. For instance, hand-rolled tobacco is much cheaper than manufactured cigarettes and if a high proportion of current smokers uses hand-rolled cigarettes, increasing tax for such product may reduce its demands. In addition, information on the history of smoking (e.g., age at initiation or duration, the length of cigarette cessation for quitters) was not available and smoking cessation was reported at baseline and at 4-year follow-up. It is therefore possible that respondents may resume smoking during or after that period. Such misclassification is more likely to underestimate smoking and mortality risks.

We acknowledge that more than $90 \%$ of current male smokers in the cohort were aged less than 45 years (and two-thirds were aged less than 35 years) when they began to be followed up in 2005. Also, the mean age at smoking initiation among male daily smokers was 17.3 years in Thailand [25]. These may explain why we did not observe a significant increase in cancer mortality (including lung cancer). More follow-up will be informative on smoking and cancer.

Smoking rates and patterns of smoking related diseases vary across regions in Thailand. For example, the Southern region $(24.6 \%)$ had the highest smoking rate in the country in 2013 followed by Northeast (22.8\%), the North (20.0\%), and Central (18.2\%) zones [43]. However, $53.5 \%$ of the new cases of lung cancer in 2009 were from Bangkok Metropolitan Area, while only 18.0 and $13.0 \%$ were from the Northeast and the South [44]. Regional differentials in association between smoking and subsequent mortality may be analysed further in the Thai Cohort Study as deaths accumulate in the future.

The implementation of tobacco control in Thailand has been recognised as a success and smoking prevalence has already reduced. The current National Tobacco Control Strategy 2012-2014 focuses on prevention of smoking initiation and reduction of current smoking [45]. Our study shows clearly the accelerated mortality associated with smoking at an early stage of the epidemic and among smokers in early middle age. The disease burden from smoking would be expected to increase rapidly among current smokers who continue to smoke and this will get worse if adolescents continue to initiate smoking at progressively earlier ages. The study also helps quantify the expected benefit for Thailand if smoking rates among males fall and smoking rates among females remain very low. Both outcomes will lead to mortality reduction due to prevention of the large set of chronic diseases attributable to smoking. Even over a short period of study including mostly young adults, we were able to detect substantial avoidable smoking related mortality. These local data provide clear evidence not previously available. As expected, the adverse effects of tobacco are very evident in Thailand; most importantly, quitting is beneficial.

\section{Abbreviations}

HR: Hazard ratios; TCS: Thai Cohort Study; CVD: Cardiovascular diseases; Cl: Confidence interval.

\section{Competing interests}

The authors declare that they have no competing interests.

\section{Authors' contributions}

JZ and VY conceptualized and drafted the manuscript with inputs from AS. JZ analysed the data. CP provided background information on smoking in Thailand. SS and AS designed and executed the Thai Health-Risk Transition project. All authors read and approved the final manuscript.

\section{Acknowledgments}

We thank the staff at Sukhothai Thammathirat Open University (STOU) who assisted with student contact, and the STOU students who are participating in the cohort study. We also thank the Thai Ministry of Interior and Ministry of Public Health for mortality investigation and cause of death data linkage.

\section{Funding}

This study was supported by the International Collaborative Research Grants Scheme with joint grants from the Wellcome Trust UK (GR071587MA) and the Australian National Health and Medical Research Council (268055), and as a global health grant from the NHMRC (585426).

\section{Author details}

${ }^{1}$ National Centre for Epidemiology and Population Health and Global Health Division, Research School of Population Health, The Australian National University, Canberra, Australia. ${ }^{2}$ International Health and Policy Program, Bureau of Policy and Strategy, Ministry of Public Health, Nonthaburi, Thailand. ${ }^{3}$ School of Human Ecology, Sukhothai Thammathirat Open University, Nonthaburi, Thailand.

Received: 17 December 2014 Accepted: 3 October 2015

Published online: 27 October 2015

\section{References}

1. Thun MJ, Carter BD, Feskanich D, Freedman ND, Prentice R, Lopez AD, et al. 50-year trends in smoking-related mortality in the United States. N Engl J Med. 2013;368(4):351-64.

2. Jha P, Jacob B, Gajalakshmi V, Gupta PC, Dhingra N, Kumar R, et al. A nationally representative case-control study of smoking and death in India. N Engl J Med. 2008;358(11):1137-47.

3. Vollset SE, Tverdal A. Gjessing HkK: Smoking and deaths between 40 and 70 years of age in women and men. Ann Intern Med. 2006;144(6):381-9.

4. Lim SS, Vos T, Flaxman AD, Danaei G, Shibuya K, Adair-Rohani H, et al. A comparative risk assessment of burden of disease and injury attributable to 67 risk factors and risk factor clusters in 21 regions, 1990-2010: a systematic analysis for the Global Burden of Disease Study 2010. Lancet. 2012;380(9859):2224-60. 
5. Levy DT, Ellis JA, Mays D, Huang AT. Smoking-related deaths averted due to three years of policy progress. Bull World Health Organ. 2013;91(7):509-18.

6. Doll R, Peto R, Boreham J, Sutherland I. Mortality in relation to smoking: 50 years' observations on male British doctors. BMJ. 2004;328(7455):1519.

7. Doll R, Peto R, Boreham J, Sutherland I. Mortality from cancer in relation to smoking: 50 years observations on British doctors. Br J Cancer. 2005;92(3):426-9.

8. Kenfield SA, Wei EK, Rosner BA, Glynn RJ, Stampfer MJ, Colditz GA. Burden of smoking on cause-specific mortality: application to the Nurses' Health Study. Tob Control. 2010;19(3):248-54.

9. Kondo T, Osugi S, Shimokata K, Honjo H, Morita Y, Maeda K, et al. Smoking and smoking cessation in relation to all-cause mortality and cardiovascular events in 25,464 healthy male Japanese workers. Circ J. 2011;75(12):2885-92.

10. Godtfredsen NS, Holst C, Prescott E, Vestbo J, Osler M. Smoking reduction, smoking cessation, and mortality: a 16-year follow-up of 19,732 men and women from The Copenhagen Centre for Prospective Population Studies. Am J Epidemiol. 2002;156(11):994-1001.

11. Sakata R, McGale P, Grant EJ, Ozasa K, Peto R, Darby SC. Impact of smoking on mortality and life expectancy in Japanese smokers: a prospective cohort study. BMJ. 2012;345, e7093.

12. Ikeda F, Ninomiya T, Doi Y, Hata J, Fukuhara M, Matsumoto T, et al. Smoking cessation improves mortality in Japanese men: the Hisayama study. Tob Control. 2012;21(4):416-21.

13. Huxley R, Jamrozik K, Lam TH, Barzi F, Ansary-Moghaddam A, Jiang CQ, et al. Asia Pacific Cohort Studies C: Impact of smoking and smoking cessation on lung cancer mortality in the Asia-Pacific region. Am J Epidemiol. 2007;165(11):1280-6.

14. Kim IS, Jee SH, Ohrr H, Yi SW. Effects of smoking on the mortality of lung cancer in Korean men. Yonsei Med J. 2001;42(2):155-60.

15. Li Y, Yamagishi K, Yatsuya H, Tamakoshi A, Iso H. Smoking cessation and COPD mortality among Japanese men and women: the JACC study. Prev Med. 2012;55(6):639-43.

16. Kupiainen $H$, Kinnula VL, Lindqvist A, Postma DS, Boezen HM, Laitinen T, et al. Successful smoking cessation in COPD: Association with comorbidities and mortality. Pulm Med. 2012;2012:725024.

17. Iso H, Date C, Yamamoto A, Toyoshima H, Watanabe Y, Kikuchi S, et al. Smoking cessation and mortality from cardiovascular disease among Japanese men and women: the JACC Study. Am J Epidemiol. 2005;161(2):170-9.

18. Asia Pacific Cohort Studies Collaboration. Smoking, quitting, and the risk of cardiovascular disease among women and men in the Asia-Pacific region. Int J Epidemiol. 2005;34(5):1036-45.

19. Honjo K, Iso H, Tsugane S, Tamakoshi A, Satoh H, Tajima K, et al. The effects of smoking and smoking cessation on mortality from cardiovascular disease among Japanese: pooled analysis of three large-scale cohort studies in Japan. Tob Control. 2010;19(1):50-7.

20. Leistikow BN, Martin DC, Jacobs J, Rocke DM, Noderer K. Smoking as a risk factor for accident death: a meta-analysis of cohort studies. Accid Anal Prev. 2000;32(3):397-405.

21. Wen CP, Tsai SP, Cheng TY, Chan HT, Chung WS, Chen CJ. Excess injury mortality among smokers: a neglected tobacco hazard. Tob Control. 2005;14 Suppl 1:i28-32

22. Tamakoshi A, Kawado M, Ozasa K, Tamakoshi K, Lin Y, Yagyu K, et al. Impact of smoking and other lifestyle factors on life expectancy among japanese: findings from the Japan Collaborative Cohort (JACC) Study. J Epidemiol; J Epidemiological Asso. 2010;20(5):370-6.

23. Thai Ministry of Public Health: Burden of Disease. Thai Health Promotion Foundation, and National Health Security Office. Nonthaburi: International Health Policy Program; 2009.

24. Pittayarangsarit S, lam-anan P, Pankrajang P, Sommitre K. Summary of tobacco control in Thailand In. Nonthaburi: Thai Health Promotion Foundation; 2011.

25. WHO. Global adult tobacco survey: Thailand report 2011, WHO regional office for south-east Asia. 2011.

26. Sleigh AC, Seubsman SA, Bain C. Cohort profile: the Thai cohort of 87,134 open university students. Int J Epidemiol. 2008;37(2):266-72.

27. Seubsman SA, Yiengprugsawan V, Sleigh AC. The Thai cohort study T: a large national Thai cohort study of the health-risk transition based on sukhothai thammathirat open university students. ASEAN J Open Distance Learn. 2012:4(1):58-69.

28. Seubsman S, Kelly M, Sleigh A, Peungson J, Chokkanapitak J, Vilainerun D. Methods used for successful follow-up in a large scale national cohort study in Thailand. BMC Res Notes. 2011;4(1):166.
29. Mathers CD, Fat DM, Inoue M, Rao C, Lopez AD. Counting the dead and what they died from: an assessment of the global status of cause of death data. Bull World Health Organ. 2005;83(3):171.

30. Prasartkul P, Vapattanawong $P$. The completeness of death registration in Thailand: evidence from demographic surveillance system of the kanchanaburi project. World Health Popul. 2006:8(3):43-51.

31. Banks E, Lim L, Seubsman SA, Bain C, Sleigh A. Relationship of obesity to physical activity, domestic activities, and sedentary behaviours: crosssectional findings from a national cohort of over 70,000 Thai adults. BMC Public Health. 2011;11:762.

32. Kanazawa M, Yoshiike N, Osaka T, Numba Y, Zimmet P, Inoue S. Criteria and classification of obesity in Japan and Asia-Oceania. World Rev Nutr Diet. 2005:94:1-12.

33. Lim LL, Seubsman SA, Sleigh A. Validity of self-reported weight, height, and body mass index among university students in Thailand: Implications for population studies of obesity in developing countries. Popul Health Metr. 2009;7.

34. Pachanee CA, Lim L, Bain C, Wibulpolprasert S, Seubsman SA, Sleigh AC. Smoking behavior among 84,315 Open University students in Thailand. Asia Pac J Public Health / Asia-Pacific Acad Consortium Public Health. 2011;23(4):544-54.

35. Thai Ministry of Public Health. Burden of Disease: Disability-Adjusted Life Year. Nonthaburi: International Health Policy Program and National Health Security Office; 2009.

36. Ueshima H, Choudhury SR, Okayama A, Hayakawa T, Kita Y, Kadowaki T, et al. Cigarette smoking as a risk factor for stroke death in Japan: NIPPON DATA80. Stroke; J Cerebral Circulation. 2004;35(8):1836-41.

37. Kamsa-Ard S, Promthet S, Lewington S, Burrett JA, Sherliker P, Kamsa-Ard S, et al. Association between smoking and mortality: Khon Kaen cohort study, Thailand. Asian Pac J Cancer Prev. 2013;14(4):2643-7.

38. Wibulpolprasert S, editor. Thailand health profile 2008-2010. Bangkok: Veteran Press; 2011.

39. Gram IT, Sandin S, Braaten T, Lund E, Weiderpass E. The hazards of death by smoking in middle-aged women. Eur J Epidemiol. 2013;28(10):799-806.

40. Singh PN, Yel D, Sin S, Khieng S, Lopez J, Job J, et al. Tobacco use among adults in Cambodia: evidence for a tobacco epidemic among women. Bull World Health Organ. 2009;87(12):905-12.

41. Jitnarin N, Kosulwat V, Rojroongwasinkul N, Boonpraderm A, Haddock CK, Poston WS. Socioeconomic status and smoking among thai adults: results of the National Thai Food Consumption Survey. Asia Pac J Public Health/ Asia-Pacific Acad Consortium Public Health. 2011;23(5):672-81.

42. Pachanee C. Smoking in Thailand: a comparison of a large national sentinel cohort to the Thai population and projected future mortality. Canberra: PhD thesis submitted to the Australian National University; 2010.

43. Punkrajang $P$, Pitayarangsarit $S$, editors. Situation of tobacco risk factor in Thailand 2014. Bangkok: Tobacco Control Research and Knowledge Management Centre; 2014.

44. National Cancer Institute. Hospital-based cancer registry 2009. Bangkok: National Cancer Institute; 2010.

45. Thai Ministry of Public Health: National Tobacco Control Strategy 2012-2014 In. http://www.hfocus.org/content/2012/06/635\#sthash.MXO0ieeZ.dpuf accessed on 15 May 2014; 2012.

\section{Submit your next manuscript to BioMed Central and take full advantage of:}

- Convenient online submission

- Thorough peer review

- No space constraints or color figure charges

- Immediate publication on acceptance

- Inclusion in PubMed, CAS, Scopus and Google Scholar

- Research which is freely available for redistribution

Submit your manuscript at www.biomedcentral.com/submit

C Biomed Central 